Efficient inverted polymer solar cells with thermal-evaporated and solution-processed small molecular electron extraction layer

Fu-Zhou Sun, Ai-Li Shi, Zai-Quan Xu, Huai-Xin Wei, Yan-Qing Li, Shuit-Tong Lee, and Jian-Xin Tang

Citation: Appl. Phys. Lett. 102, 133303 (2013); doi: 10.1063/1.4799833

View online: http://dx.doi.org/10.1063/1.4799833

View Table of Contents: http://aip.scitation.org/toc/apl/102/13

Published by the American Institute of Physics

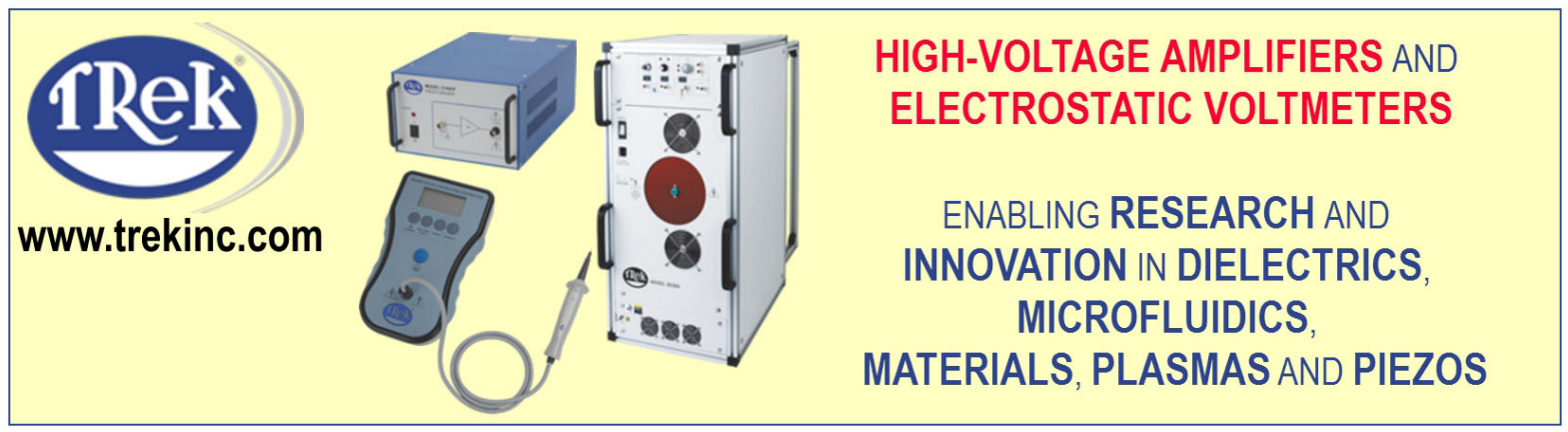




\title{
Efficient inverted polymer solar cells with thermal-evaporated and solution-processed small molecular electron extraction layer
}

\author{
Fu-Zhou Sun, Ai-Li Shi, Zai-Quan Xu, Huai-Xin Wei, Yan-Qing Li, ${ }^{\text {a) }}$ Shuit-Tong Lee, \\ and Jian-Xin Tang ${ }^{\text {a) }}$ \\ Jiangsu Key Laboratory for Carbon-Based Functional Materials \& Devices, Institute of Functional Nano \& \\ Soft Materials (FUNSOM), Soochow University, Suzhou 215123, China
}

(Received 14 February 2013; accepted 21 March 2013; published online 5 April 2013)

\begin{abstract}
Efficient inverted polymer solar cell is reported upon by integrating with a small molecular 1,3,5tri(phenyl-2-benzimi-dazolyl)-benzene (TPBi) electron extraction layer (EEL) at low processing temperature with thermal-evaporation and solution-process, resulting in the power conversion efficiencies of $3.70 \%$ and $3.47 \%$, respectively. The potential of TPBi as an efficient EEL is associated with its suitable electronic energy level for electron extraction and hole blocking from the active layer to the indium tin oxide cathode. (c) 2013 American Institute of Physics. [http://dx.doi.org/10.1063/1.4799833]
\end{abstract}

Polymer solar cells (PSCs) have drawn considerable attention in recent years due to their advantages of low cost, light-weight, simple solution processing, and mechanical flexibility for special substrates. ${ }^{1-4}$ Currently, major challenges for the commercial success of PSCs are the achievement of competitive power conversion efficiency (PCE) and the demonstration of long-term air stability. ${ }^{5-7} \mathrm{~A}$ typical PSC is fabricated with a structure of a transparent conductive anode (e.g., indium tin oxide, ITO), a low work function (WF) metal cathode (e.g., Al, Ca), and a blended conjugated polymer/fullerene derivative active layer sandwiched between these two electrodes. However, low WF metallic cathode is susceptible to be oxidized upon exposure to oxygen and water vapor, which can seriously lead to the degradation of PSCs. ${ }^{8}$ In addition, it has been demonstrated recently that vertical phase separation spontaneously occurs in polymer active layer with the fullerene derivative rich adjacent to the substrate interface. ${ }^{9,10}$ To address these challenges in the regular structure devices, inverted PSCs have been developed as a promising alternative for obtaining high device performance, in which ITO is used as the bottom cathode to collect electrons instead of the top metal electrode. ${ }^{11}$

It has been recognized that one important factor to the cell performance of inverted PSCs is an efficient electron extraction layer (EEL) incorporated between ITO cathode and the active layer, which can tremendously promote the efficient extraction of electrons and accordingly the photovoltaic performance of PSCs. Tremendous efforts have been made for the development of EELs in inverted PSCs. ${ }^{12-19}$ As reported in the literature, inorganic EEL materials, such as cesium carbonate $\left(\mathrm{Cs}_{2} \mathrm{CO}_{3}\right),{ }^{16} \mathrm{ZnO},{ }^{17} \mathrm{TiOx},{ }^{18}$ and $\mathrm{Al}_{2} \mathrm{O}_{3},{ }^{19}$ have been widely utilized to achieve a remarkable device performance due to their large band gaps and good electron extraction property. However, a relatively high-temperature annealing process during fabrication process and the brittle nature of inorganic EEL will inevitably inhibit their application with the flexible substrates. Organic materials are a

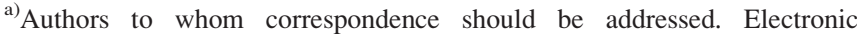
addresses: jxtang@suda.edu.cn and yqli@suda.edu.cn.
}

promising alternative as efficient EELs because of their great mechanical flexibility and the ease of fabrication process with controlled film morphology and chemical composition at low processing temperature. Recently, some attempts have been made for using small molecules or polymers as EELs in inverted PSCs. ${ }^{20-24}$

In this Letter, the potential utilization of n-type widegap small molecule of 1,3,5-tri(phenyl-2-benzimi-dazolyl)benzene (TPBi) as an EEL in inverted PSCs is explored, which can be easily fabricated by thermal evaporation and solution processing. The selection of TPBi as an EEL for inverted PSC arises from its easy thermal deposition, preferable solubility, superior exciton/hole blocking capability, highly optical transparency with large band gap, and relatively high electron mobility. ${ }^{25}$ A correlation between cell response parameters and the film properties of TPBi layer is investigated. Using data on interface energetics and electrical properties, it is shown that both vacuum deposited and spin-coated TPBi layers can form a suitable energy-level contact between the active layer and ITO cathode for electron extraction.

All chemicals were used as received. The device structure of inverted PSCs and molecule structure of TPBi are shown in the insets of Figs. 1(a) and 1(b), respectively. Prior to device fabrication, the patterned ITO glass substrates with a sheet resistance of $20 \Omega / \mathrm{sq}$ were treated upon a routine cleaning process. Then, the TPBi film was thermally deposited in a vacuum deposition chamber with a base pressure of $2 \times 10^{-6}$ Torr, whose thickness was controlled by quartz oscillating crystal monitor. For the solution-processed TPBi film, the precursor solution was prepared with solvents of chloroform, methanoic acid, or methanol:ethanol mixture (weight ratio of 1:3) by a TPBi weight ratio of $0.375 \%$. The solution-processed TPBi film was spin-coated onto ITO substrate in nitrogen $\left(\mathrm{N}_{2}\right)$-filled glove box, and then immediately annealed at $50^{\circ} \mathrm{C}$ for $20 \mathrm{~min}$. The thicknesses of TPBi films prepared with different methods were verified by an alpha$\mathrm{SE}^{\mathrm{M}}$ Spectroscopic Ellipsometer. After the preparation of TPBi films, the resulting samples were spin-coated with the blend of poly(3-hexylthiophene):[6,6]-phenyl C61 butyric acid methyl ester (P3HT:PCBM) with a 1:0.8 weight ratio 
$(10 \mathrm{mg} / \mathrm{ml}$, dissolved in dichlorobenzene) at $600 \mathrm{rpm}$ for $1 \mathrm{~min}$. The wet film was dried at room temperature for $30 \mathrm{~min}$, and then annealed on a hot plate at $110^{\circ} \mathrm{C}$ for $10 \mathrm{~min}$ inside the glove box. The thickness of the resultant P3HT:PCBM blend film was determined to be approximately $110 \mathrm{~nm}$. Finally, the devices were completed by thermally evaporating an $8 \mathrm{~nm}$-thick $\mathrm{MoO}_{3}$ layer as a hole collection layer and a $100 \mathrm{~nm}$-thick $\mathrm{Al}$ anode through shadow masks in the vacuum deposition chamber. The active device area was estimated to be $0.1 \mathrm{~cm}^{2}$ as determined by the overlap of ITO cathode and $\mathrm{Al}$ anode.

Photovoltaic measurements of inverted PSCs were carried out in ambient condition without encapsulation. The experimental setup has been described elsewhere. ${ }^{12,20}$ The electronic structures of the films were determined by ultraviolet photoelectron spectroscopy (UPS) in a Kratos AXIS Ultra-DLD ultrahigh vacuum system (a base pressure of $3 \times 10^{-10}$ Torr) with HeI excitation (21.2 eV for UPS). ${ }^{26}$

Figure 1(a) plots the current density-voltage (J-V) characteristics of inverted PSCs under an air mass (AM) $1.5 \mathrm{G}$ irradiation of $100 \mathrm{~mW} / \mathrm{cm}^{2}$ as a function of film thicknesses of TPBi
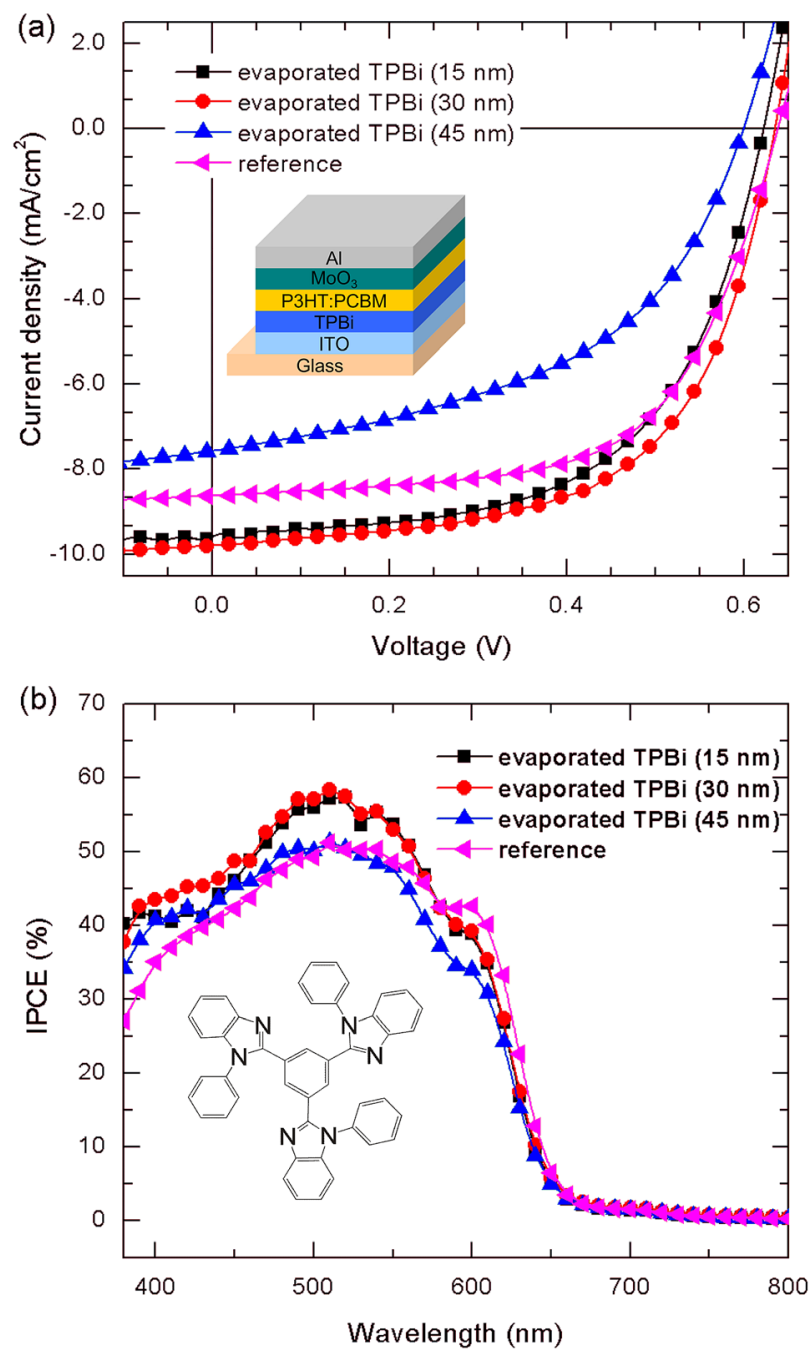

FIG. 1. (a) J-V characteristics of inverted PSCs integrated with various thickness TPBi EEL under $100 \mathrm{~mW} / \mathrm{cm}^{2}$ illumination. Inset: structure schematic of inverted PSCs. (b) The corresponding IPCE spectra. Inset: chemical structure of TPBi molecule. The reference device with a structure of ITO/ PEDOT:PSS/P3HT:PCBM/LiF/Al is also shown for comparison.
EEL ranging from $15 \mathrm{~nm}$ to $45 \mathrm{~nm}$. For comparison, a reference device with a regular structure of ITO/poly (3, 4-ethylenedioxythiophene):(polystyrene sulfonic acid) (PEDOT:PSS) $(40 \mathrm{~nm}) / \mathrm{P} 3 \mathrm{HT}: \mathrm{PCBM}(110 \mathrm{~nm}) / \mathrm{LiF}(0.5 \mathrm{~nm}) / \mathrm{Al}(100 \mathrm{~nm})$ was also fabricated with the identical process parameters during the same batch processing, where the PEDOT:PSS layer was used as a buffer layer for hole extraction. The corresponding photovoltaic parameters are summarized in Table I. As depicted in Fig. 1(a), inverted PSCs with the incorporation of a TPBi EEL show a significant dependence on TPBi layer thickness. The device with an optimal TPBi EEL of $30 \mathrm{~nm}$ thick yields the best device performance, which exhibits a PCE of $3.70 \%$ with an open-circuit voltage $\left(\mathrm{V}_{\mathrm{OC}}\right)$ of $0.63 \mathrm{~V}$, a fill factor $(\mathrm{FF})$ of $60 \%$, a short-circuit current density $\left(\mathrm{J}_{\mathrm{SC}}\right)$ of $9.80 \mathrm{~mA} / \mathrm{cm}^{2}$, and a series resistance $\left(\mathrm{R}_{\mathrm{S}}\right)$ of $25 \Omega \mathrm{cm}^{2}$, respectively. For comparison, the reference PSC shows a lower PCE of 3.37\%. The results indicate that a TPBi layer can provide the functionality for electron extraction and transport from the electron-acceptor material to ITO cathode. In addition, the dependence of the resultant device performance on TPBi layer thickness is well matched with the trend of $R_{S}$. The increased Rs can lead to a reduction in $\mathrm{J}_{\mathrm{SC}}, \mathrm{FF}$, and $\mathrm{V}_{\mathrm{OC}}$, hence a reduction in PCE.

To get additional insight into device performance, Fig. 1(b) compares the incident photon to current conversion efficiency (IPCE) spectra of the corresponding inverted PSCs shown in Fig. 1(a). The difference in shape of IPCE spectra is negligible for inverted devices with a TPBi EEL, while their intensity are consistent with the J-V properties as plotted in Fig. 1(a). It is, therefore, considered that the performance enhancement for inverted PSCs is associated with the influence of TPBi layer instead of the effect of P3HT:PCBM active layer.

Compared to thermal evaporation, a solution process has giant advantage on the high throughput, large-area, and low-cost fabrication processes of PSCs. Therefore, it is highly desired to produce high-performance inverted PSCs by solution-based fabrication process for TPBi layer. A suitable solvent is essential to realize a solution-processed TPBi film with good film property, which can form a good contact with the active layer and ITO substrate. Here, the TPBi precursor solutions were prepared by various solvents, including chloroform, methanoic acid, and methanol:ethanol mixture. As summarized in Table I, the device with a TPBi precursor solution prepared by a methanol:ethanol mixture shows the best performance with a PCE of $3.47 \%$, which is similar to that of thermal-evaporated TPBi devices.

TABLE I. Photovoltaic characteristics of inverted PSCs with a configuration of ITO/TPBi/P3HT:PCBM/ $\mathrm{MoO}_{3} / \mathrm{Al}$. The reference device with a structure of ITO/PEDOT:PSS/P3HT:PCBM/LiF/Al is also shown for comparison.

\begin{tabular}{lccccc}
\hline \hline TPBi layer & $\begin{array}{l}\mathrm{V}_{\mathrm{OC}} \\
(\mathrm{V})\end{array}$ & $\begin{array}{c}\mathrm{J}_{\mathrm{SC}} \\
\left(\mathrm{mA} / \mathrm{cm}^{2}\right)\end{array}$ & $\begin{array}{c}\mathrm{FF} \\
(\%)\end{array}$ & $\begin{array}{c}\mathrm{PCE} \\
(\%)\end{array}$ & $\begin{array}{c}\mathrm{R}_{\mathrm{S}} \\
\left(\Omega \mathrm{cm}^{2}\right)\end{array}$ \\
\hline Reference & 0.64 & 8.62 & 61 & 3.37 & 62 \\
Evaporation, 15 nm & 0.62 & 9.53 & 58 & 3.43 & 34 \\
Evaporation, 30 nm & 0.63 & 9.80 & 60 & 3.70 & 25 \\
Evaporation, 45 nm & 0.59 & 7.56 & 49 & 2.19 & 54 \\
Chloroform & 0.37 & 9.83 & 45 & 1.64 & 102 \\
Methanoic acid & 0.55 & 10.49 & 54 & 3.12 & 36 \\
Methanol:ethanol & 0.57 & 10.67 & 57 & 3.47 & 28 \\
\hline \hline
\end{tabular}


To identify the key factors that govern the device performance of inverted PSCs, optical, morphological, and electronic properties of the TPBi layers was examined. The absorption and transmittance spectra of thermal-evaporated and solution-processed TPBi layers (not shown here) were measured by an UV/vis/near-IR spectrometer (Perkin Elmer Lambda 750), exhibiting high transmittance in the visible range from $400 \mathrm{~nm}$ to $650 \mathrm{~nm}$. The high transparency of TPBi layers with different formation methods is due to the large energy gaps of 3.3 and $3.5 \mathrm{eV}$, respectively, for thermal-evaporated and solution-processed TPBi layers. It indicates that TPBi layers have no impact on the incident light loss delivered to the active layer under illumination.

It is known that the formation of P3HT:PCBM active layer is rather sensitive to the underlying interfacial layer, and an important factor influencing the performance of these devices is the interfacial morphology properties. ${ }^{27}$ Thus, the topography and surface roughness of TPBi layers and P3HT:PCBM blend on top of different TPBi layers were examined by atomic force microscopy (AFM) (Veeco MultiMode V) in tapping mode. Figures 2(a) and 2(b) display the surface morphologies of TPBi layer prepared by thermal-evaporation and solution-process on ITO substrates. It is clear that the surfaces of both layers are smooth and contiguous with the root mean square roughness values of 1.41 and $0.51 \mathrm{~nm}$ for thermal evaporation and solution process, respectively. Figures 2(c) and 2(d) show surface morphologies of the P3HT:PCBM active layer spin-coated on thermalevaporated and solution-processed TPBi films, respectively, where uniform and smooth films are formed with negligible difference in morphology. It implies that the formation methods of TPBi layers have no significant influence on the film morphology of P3HT:PCBM blend.

The electronic structures at the interface for P3HT:PCBM spin-coated on thermal-evaporated and solution-processed TPBi layers were examined by UPS measurement, where the methanol:ethanol (1:3) was used for the solvent. Based on the UPS data, schematic energy level alignments of the component
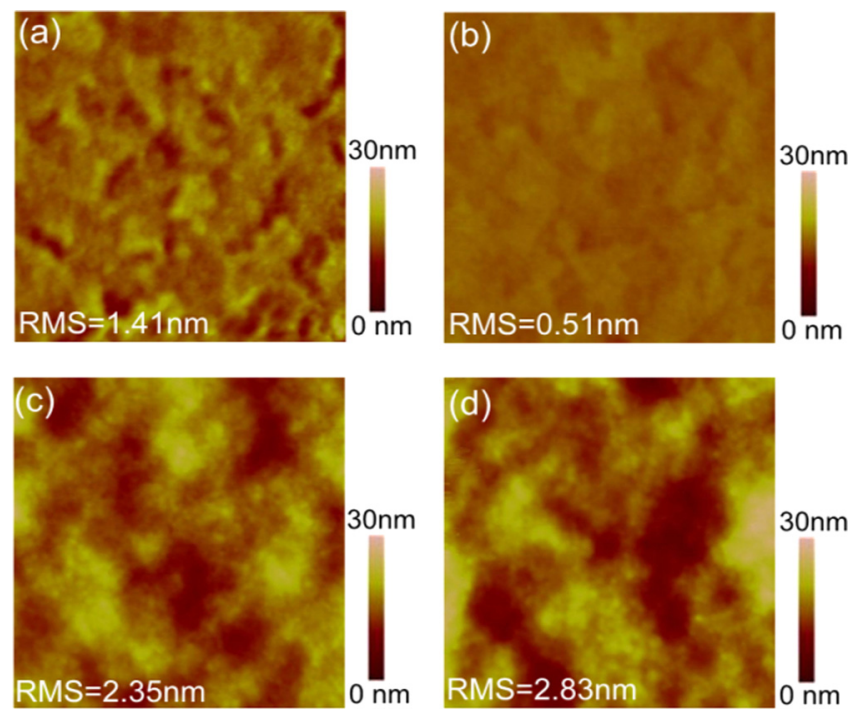

FIG. 2. $2 \mu \mathrm{m} \times 2 \mu \mathrm{m}$ AFM images of (a) evaporated TPBi and (b) solution processed TPBi films on ITO substrate. Surface morphologies of spin-coated P3HT:PCBM films on (c) evaporated TPBi and (d) solution processed TPBi.

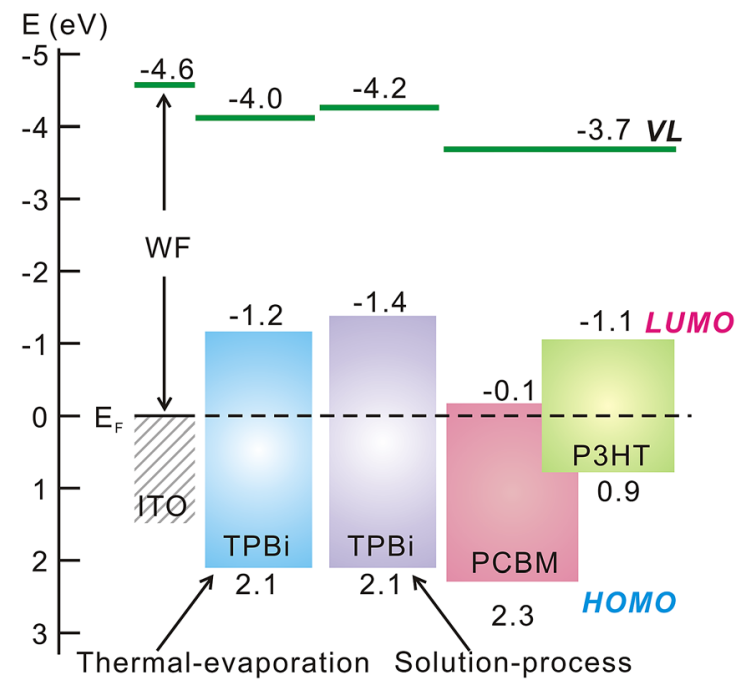

FIG. 3. Energy level diagrams of EEL/P3HT:PCBM active layer in inverted PSCs with TPBi prepared by thermal evaporation and solution processing.

materials used in the inverted configuration can be accurately determined with the Fermi level $\left(\mathrm{E}_{\mathrm{F}}\right)$ alignment as illustrated in Fig. 3. Here, the WF and highest occupied molecular orbital (HOMO) values relative to $\mathrm{E}_{\mathrm{F}}$ are directly extracted from the UPS spectra (not shown here). The lowest unoccupied molecular orbital (LUMO) positions are derived by adding the energy gaps, which are obtained from absorption meausurement. ${ }^{20}$ However, it should be mentioned that the optical energy gaps may underestimate the LUMO position. The WF values of thermal-evaporated and solution-processed TPBi layers on ITO substrate are $4.0 \mathrm{eV}$ and $4.2 \mathrm{eV}$, respectively. Therefore, the LUMOs of thermal-evaporated and solution-processed TPBi layers are estimated to be 1.2 and $1.4 \mathrm{eV}$ above $\mathrm{E}_{\mathrm{F}}$. The energy levels of PCBM and P3HT in the P3HT:PCBM blend are aligned with a common vacuum level (VL), while there is a VL shift at the TPBi/P3HT:PCBM interface as determined by UPS measurement. As shown in Fig. 3, the wide energy gap of TPBi layer causes a straddling type of HOMO and LUMO offsets between TPBi layer and the P3HT:PCBM active layer, which is indicative of efficient hole/exciton blocking from the P3HT:PCBM active layer to ITO cathode. In addition, it is shown in Table I that the device with solution-processed TPBi layer by methanol:ethanol solvent exhibits a $\mathrm{V}_{\mathrm{OC}}$ of $0.57 \mathrm{~V}$, which is lower than that using a thermal-evaporated TPBi (i.e., $0.63 \mathrm{~V})$. Such a discrepancy is clearly due to the difference in LUMO offsets between two TPBi and PCBM as illustrated in Fig. 3, where the thermal evaporated TPBi can form a lower energy barrier with the P3HT:PCBM active layer for electron extraction.

In summary, efficient inverted PSCs have been demonstrated by incorporating a small molecular TPBi layer for electron extraction, in which the PCEs with thermalevaporated and solution-processed TPBi EELs are 3.70\% and $3.47 \%$, respectively. The potential of TPBi as an efficient EEL is associated with its suitable electronic energy level for electron extraction and hole blocking from the active layer to the ITO cathode.

The authors acknowledge the financial support by NSFC (Nos. 61007020, 91027041, 61107022, and 61036009), 
Jiangsu Science and Technology Department (No. BK2011280), Bureau of Science and Technology of Suzhou Municipality (Nos. SYG201237 and SYG201232), and PAPD.

${ }^{1}$ G. Dennler, M. C. Scharber, and C. J. Brabec, Adv. Mater. 21, 1323 (2009).

${ }^{2}$ P. W. M. Blom, V. D. Mihailetchi, L. J. A. Koster, and D. E. Markov, Adv. Mater. 19, 1551 (2007).

${ }^{3}$ J. Chen and Y. Cao, Acc. Chem. Res. 42, 1709 (2009).

${ }^{4}$ L. T. Dou, J. B. You, J. Yang, C. C. Chen, Y. J. He, S. Murase, T. Moriarty, K. Emery, G. Li, and Y. Yang, Nat. Photonics 6, 180 (2012).

${ }^{5}$ W. J. Potscavage, Jr., A. Sharma, and B. Kippelen, Acc. Chem. Res. 42, 1758 (2009).

${ }^{6}$ H. Ma, H. L. Yip, F. Huang, and A. K.-Y. Jen, Adv. Funct. Mater. 20, 1371 (2010).

${ }^{7}$ Z. Q. Xu, J. Li, J. P. Yang, P. P. Cheng, J. Zhao, S. T. Lee, Y. Q. Li, and J. X. Tang, Appl. Phys. Lett. 98, 253303 (2011).

${ }^{8}$ C. Y. Li, T. C. Wen, T. H. Lee, T. F. Guo, J. C. A. Huang, Y. C. Lin, and Y. J. Hsu, J. Mater. Chem. 19, 1643 (2009).

${ }^{9}$ Z. Xu, L. M. Chen, G. W. Yang, C. H. Huang, J. H. Hou, Y. Wu, G. Li, C. S. Hsu, and Y. Yang, Adv. Funct. Mater. 19, 1227 (2009).

${ }^{10}$ M. Campoy-Quiles, T. Ferenczi, T. Agostinelli, P. G. Etchrgoin, Y. Kim, T. D. Anthopoulos, P. N. Stavrinou, D. D. C. Bradley, and J. Nelson, Nature Mater. 7, 158 (2008).

${ }^{11}$ S. Sista, M. H. Park, Z. R. Hong, Y. Wu, J. H. Hou, W. L. Kwan, G. Li, and Y. Yang, Adv. Mater. 22, 380 (2010).

${ }^{12}$ J. J. Zhu, Z. Q. Xu, G. Q. Fan, S. T. Lee, Y. Q. Li, and J. X. Tang, Org. Electron. 12, 2151 (2011).
${ }^{13}$ T. Kuwabara, Y. Kawahara, T. Yamaguchi, and K. Takahashi, Appl. Mater. Interfaces 1, 2107 (2009).

${ }^{14}$ J. Gilot, I. Barbu, M. M. Wienk, and R. A. J. Janssen, Appl. Phys. Lett. 91, 113520 (2007).

${ }^{15}$ S. K. Hau, H. L. Yip, and A. K. Jen, Polym. Rev. 50, 474 (2010).

${ }^{16}$ G. Li, C. W. Chu, V. Shrotriya, J. Huang, and Y. Yang, Appl. Phys. Lett. 88, 253503 (2006).

${ }^{17}$ C. Waldauf, M. Morana, P. Denk, P. Schilinsky, K. Coakley, S. A. Choulis, and C. J. Brabec, Appl. Phys. Lett. 89, 233517 (2006).

${ }^{18}$ M. S. White, D. C. Olson, S. E. Shaheen, N. Kopidakis, and D. S. Ginley, Appl. Phys. Lett. 89, 143517 (2006).

${ }^{19}$ Y. H. Zhou, H. Cheun, W. J. Potscavage, C. Fuentes-Hernandez, S. J. Kim, and B. Kippelen, J. Mater. Chem. 20, 6189 (2010).

${ }^{20}$ Z. Q. Xu, J. P. Yang, F. Z. Sun, S. T. Lee, Y. Q. Li, and J. X. Tang, Org. Electron. 13, 697 (2012).

${ }^{21}$ Z. C. He, C. M. Zhong, S. J. Su, M. Xu, H. B. Wu, and Y. Cao, Nat. Photonics 6, 591 (2012).

${ }^{22}$ Z. A. Tan, W. Q. Zhang, Z. G. Zhang, D. P. Qian, Y. Huang, J. H. Hou, and Y. F. Li, Adv. Mater. 24, 1476 (2012).

${ }^{23}$ S. I. Na, T. S. Kim, S. H. Oh, J. Kim, S. S. Kim, and D. Y. Kim, Appl. Phys. Lett. 97, 223305 (2010).

${ }^{24}$ Y. H. Zhou, F. H. Li, S. Barrau, W. J. Tian, O. Inganas, and F. L. Zhang, Sol. Energy Mater. Sol. Cells 93, 497 (2009).

${ }^{25}$ Y. Q. Li, M. K. Fung, Z. Y. Xie, S. T. Lee, L. S. Hung, and J. M. Shi, Adv. Mater. 14, 1317 (2002).

${ }^{26}$ J. P. Yang, Y. Xiao, Y. H. Deng, S. Duhm, N. Ueno, S. T. Lee, Y. Q. Li, and J. X. Tang, Adv. Funct. Mater. 22, 600 (2012).

${ }^{27}$ X. Bulliard, S. G. Ihn, S. Yun, Y. Kim, D. Choi, J. Y. Choi, M. Kim, M. Sim, J. H. Park, W. Choi, and K. Cho, Adv. Funct. Mater. 20, 4381 (2010). 